\title{
La Didáctica en la Investigación Administrativa en la Educación Superior, desde un Enfoque Sociocultural
}

\author{
A sociocultural approach to didactics in administrative research in \\ higher education
}

Armando Sánchez-Macías ${ }^{1}$
José Zilberstein Toruncha $^{2}$
Virginia Azuara Pugliese $^{3}$

\section{RESUMEN}

Ante una nueva realidad basada en avances tecnológicos, globalización y cambios en la sociedad del conocimiento, la Pedagogía y la Didáctica deben transformarse. El objetivo de este artículo es presentar la caracterización de principios y prácticas del Modelo Pedagógico y la Concepción Didáctica en la Investigación Administrativa, a través de la revisión teórica contextualizada. Planteando lineamientos en el proceso enseñanza-aprendizaje, proponiendo al Diagnóstico Organizacional y las propuestas basadas en la Administración Estratégica, como los ejes didácticos en la Investigación Administrativa. Las Instituciones de Educación Superior, deben asumir el compromiso y la responsabilidad de convertirse en formadores de recursos humanos comprometidos con la sociedad, formando profesionales competentes a través de una educación retomando la perspectiva Sociocultural.

Palabras-clave: Investigación administrativa, modelo pedagógico, didáctica, diagnóstico organizacional, educación superior, enfoque sociocultural.

\begin{abstract}
In the presence of a new reality based on technological advances, globalization and changes in the knowledge society, Pedagogy and Didactics must be transformed. The objective of this article is to present the characterization of principles and practices of the Pedagogical Model and the Didactic Conception in the Administrative Investigation, through the contextualized theoretical revision. Raising guidelines in the teaching-learning process. The Organizational Diagnosis and the Strategic Administration are proposed, as the didactic axes in the Administrative Investigation. The Institutions of Higher Education must assume the commitment and responsibility to become personnel developers committed to society, training competent professionals through an education from a Sociocultural perspective.
\end{abstract}

Keywords: Administrative research, pedagogical model, didactics, organizational diagnosis, higher education.

1 Doctor en Educación. Profesor Investigador de la Coordinación Académica Región Altiplano Oeste. Universidad Autónoma de San Luis Potosí, México. armando.sanchez@uaslp.mx

2 Doctor en Ciencias Pedagógicas. Director del Campus Tequis de la Universidad Tangamanga, México. jzilberstein@utan.edu.mx

3 Doctora en Mercadotecnia. Coordinadora de la Licenciatura en Administración en la Coordinación Académica Región Altiplano Oeste. Universidad Autónoma de San Luis Potosí, México. virginia.azuara@uaslp.mx 


\section{Introducción}

Las universidades se encuentran en un nuevo contexto de un mundo globalizado en el que, deben transformar sus estructuras internas (UNESCO, 2005) para algo más que enseñar. Dicho proceso implica un replanteamiento de sus actividades, de modo que se abra un espacio importante para la investigación. La modernidad ha cambiado la concepción actual de las Instituciones de Educación Superior (IES).

La educación universitaria se polariza hacia dos visiones. La capitalista basada en la sociedad de masas, apuntalada en los pilares de la cultura comercial, la sociedad de consumo y la institución publicitaria (ACOSTA, 2014). Por otro lado, aquella que tiende hacia la universalización, formadora de valores naturales y comunes a todas las personas, de cualquier tradición y cultura. (ZILBERSTEIN Y OLMEDO, 2015; ZILBERSTEIN, SILVESTRE Y OLMEDO, 2016).

Los ambientes de aprendizaje se han multiplicado y tienden a desarrollar una forma extendida de autoaprendizaje (DIDRIKSSON, 2004).

El nuevo conocimiento ya no sólo se genera en los ambientes universitarios o académicos, las empresas e instituciones son ahora fuertes actores.

Así mismo, el mercado laboral quedó lejos de ser el referente para la movilidad, el autodesarrollo de grupos, redes y equipos de trabajo multi e interdisciplinarios ha cobrado un gran auge (DE LA GARZA TOLEDO, 2010).

El conocimiento es un producto de la actividad y del trabajo humano (LEONTIEV, 1972; 1982), por lo tanto, su calidad está ligada a la organización y dirección de los procesos de conducción de las actividades que se realizan para la producción, difusión y aplicación de este en la sociedad (BOISIER, 2016). Así pues, los nuevos modelos de investigación y gestión ya no son propuestos únicamente por las universidades, existen nuevos actores a la altura y a la vanguardia en diversos campos del conocimiento, la ciencia y la tecnología.

Dadas las nuevas condiciones mencionadas, la Pedagogía y la Didáctica deben adaptarse a los retos y realidades que se presentan en la actualidad 
(ZILBERSTEIN Y OLMEDO, 2015). El objetivo del presente artículo es presentar una caracterización de los principios y prácticas de un Modelo Pedagógico y la Concepción Didáctica para la Investigación Administrativa que fueron investigados en el contexto de la educación superior en México entre los años 2010 y 2013 (SÁNCHEZ MACÍAS, 2014).

\section{Modelo Pedagógico para la Investigación Administrativa}

El Modelo Pedagógico que se investigó (SÁNCHEZ MACÍAS, 2014) considera a la enseñanza y el aprendizaje como un proceso de base profundamente cultural, de naturaleza histórica y social (ZANKOV, 1984; VIGOTSKY, 2013; ZILBERSTEIN Y OLMEDO, 2015), en correspondencia con el área del conocimiento, en este caso la Ciencia Administrativa. Debe ser intensamente reflexivo, a partir de la construcción y apropiación del conocimiento, habilidades y valores por parte del estudiante, lo que garantiza la implicación plenamente consciente en su propia formación permanente, como protagonista de esta.

Se retomaron como una de las bases teóricas fundamentales para la Construcción del Modelo, los Principios Didácticos para un proceso de enseñanza aprendizaje desarrollador experimentados por Silvestre y Zilberstein (2004, p. 5357), Zilberstein (2004) estos son:

- Diagnóstico Integral de la preparación del alumno para las exigencias del proceso de enseñanza aprendizaje, nivel de logros y potencialidades en el contenido de aprendizaje, desarrollo intelectual y afectivo valorativo.

- Estructurar el proceso de enseñanza aprendizaje hacia la búsqueda activa del conocimiento por el alumno, teniendo en cuenta las acciones a realizar por este en los momentos de orientación, ejecución y control de la actividad.

- Estimular la formación de conceptos y el desarrollo de los procesos lógicos de pensamiento, y el alcance del nivel teórico, en la medida que se produce la apropiación de los conocimientos y se eleva la capacidad de resolver problemas.

- Desarrollar formas de actividad y de comunicación colectivas, que favorezcan el desarrollo intelectual, logrando la adecuada interacción de lo individual con 
lo colectivo en el proceso de aprendizaje.

- Atender las diferencias individuales en el desarrollo de los escolares, en el tránsito del nivel logrado hacia el que se aspira.

- Vincular el contenido de aprendizaje con la práctica social y estimular la valoración por el alumno en el plano educativo.

El Modelo se construyó utilizando métodos de carácter teórico: como: análisis-síntesis, análisis histórico-lógico, ascenso de los abstracto a lo complejo y modelación teórica y finalmente se sometió a la valoración mediante expertos y un estudio de casos en la Universidad Tangamanga de San Luís Potosí, México (SÁNCHEZ MACÍAS, 2014).

Este Modelo considera al docente como "un educador que incentiva, potencia, promueve el desarrollo de la actividad independiente en la búsqueda de nuevos conocimientos, favorece el surgimiento de nuevas y variadas motivaciones e intereses personales, la formación de valores, de sentimientos, que en sentido general promueve la formación de la cultura" (ZILBERSTEIN; SILVESTRE Y OLMEDO, 2016).

Los preceptos que permiten al docente -en este caso en el rol de profesor de investigación, asesor o director de tesis- conducir al alumno hacia el desarrollo integral en su contexto histórico-lógico son definidos por Zilberstein (2004) y Zilberstein y Olmedo $(2014 ; 2015)$ como principios didácticos: “ $[\ldots]$ aquellas regularidades esenciales que rigen el enseñar y el aprender, que permiten al educador dirigir científicamente el desarrollo integral de la personalidad de las alumnas y alumnos". (ZILBERSTEIN, 2004, p. 107).

Estas condiciones, acciones y operaciones son las que el docente debe propiciar como preliminares y concurrentes del Proceso de Enseñanza Aprendizaje Desarrollador (PEAD) con el estudiante de educación superior en el área de Administración. La finalidad es el desarrollo de competencias investigativas que llevarán a elaborar propuestas que permitan ofrecer alternativas de solución a problemáticas de la realidad administrativa en que se desempeñan los estudiantes de los posgrados de Administración, a la vez que se formen como profesionistas que apliquen a su trabajo cotidiano, la actividad científica. 
En estos lineamientos, al estudiante se le ha denominado alumnoinvestigador, con la intención de insistir y clarificar su naturaleza: una persona que está incursionando por primera vez en la investigación, sin antecedentes ni experiencia formal en ella y sin conocimientos metodológicos previos, en la mayoría de los casos.

A partir de la revisión teórica y empírica, en el presente trabajo se proponen los principios para el proceso enseñanza aprendizaje de la investigación en el área de administración. Estos Principios se presentan a continuación:

\section{a) El alumno-investigador es un ente social formado a partir de su} entorno y contexto histórico cultural. El resultado de $\mathrm{su}$ trabajo investigativo debe tener como propósito servir a su comunidad y a la sociedad en general. La investigación, como se estableció, es el camino que contribuye al conocimiento científico de la humanidad, la enriquece y alimenta. ¿Es el conocimiento la finalidad de los procesos educativos? Los autores consideran que dicha afirmación es errónea. La producción de conocimiento administrativo no puede ser el fin último de los programas de esta área. El conocimiento es un medio que sirve para resolver problemas y mejorar las condiciones de las personas que integran la sociedad y las organizaciones. Por lo tanto, en este trabajo se establece como fin último e inalterable del proceso de investigación y del conocimiento al ser humano, su beneficio, crecimiento y desarrollo. No son las tesis terminadas y defendidas la pretensión que debe buscarse a toda costa, sino el crecimiento y desarrollo integral del ser humano (en este caso, del alumnoinvestigador). Más que crecimiento, evolución, para que el conocimiento no lo envanezca, sino por lo contrario, lo haga humilde y consciente de que el hecho de haber recibido una educación implica un compromiso con la sociedad. En este sentido, el asesor de tesis debe favorecer esta postura filosófica desde su práctica personal para poderla transmitir a sus alumnos mediante el ejemplo, es decir, de manera vivencial. Luego, el asesor de tesis transmite valores

[...] cuando potencia el espíritu crítico a sus alumnos, los provoca, les da puntos de reflexión, cuando no da tratos discriminatorios, cuando expresa tolerancia en clase, cuando fomenta hábitos de estudio y, en definitiva, conecta con las necesidades de sus alumnos (GUTIÉRREZ, 1992, p. 99). 
b) El alumno-investigador tiene su propio marco de referencia sociocultural.

El docente para el logro de resultados debe distanciarse de la perspectiva desde la cual se considera él mismo y al alumno como recurso humano. Las personas no son recursos, el razonamiento, cognición e ideas, si bien pueden ponerse al servicio de un proyecto u organización le pertenecen de manera inalienable al sujeto, formado históricamente. Sólo el trabajo puede ser considerado como recurso y éste es una consecuencia de la acción humana (LEONTIEV, 1972, 1982).

El ser humano existe en su circunstancia y nunca fuera de ella (ORTEGA Y GASSET, 2004). La toma de consciencia de su contexto histórico social es la que permite al hombre transcender de la esencia a la existencia y por ende su realidad. Tal escenario debe ser abordado por el docente asumiendo una postura comprensiva, empática y amistosa. El docente debe conducir el proceso a través de la receptividad, negociación, discusión guiada, apertura a otros puntos de vista, clarificación de malentendidos (DÍAZ Y HERNÁNDEZ, 2015). Entender la actividad del alumno desde su contexto histórico y cultural, y actuar en consecuencia es fundamental para lograr la educación integral a la que se aspira y que es el camino que se postula para lograr resultados satisfactorios.

c) El asesor de tesis debe tener en cuenta que el marco histórico y cultural del alumno investigador y el de él mismo tiende a subjetivizar el proceso, lo cual debe ser tomado en cuenta para buscar resultados científicos, basados en hechos comprobados y comprobables, sin dejar de tener en cuenta la subjetividad de procesos sociales que son importantes tener en cuenta.

La conciencia de que la investigación es situacional y ligada indefectiblemente a las circunstancias históricas, políticas, económicas, sociales y personales. Esto implica que se debe evaluar y monitorear en contexto para no desconocer las dinámicas locales y globales en las que está inserta la organización objeto de estudio. A la vez, EL asesor de tesis debe propiciar que el 
alumno defina el problema científico y el tema de manera integral y no como un hecho aislado de la realidad, de preferencia debe estar vinculado al entorno en el cual desarrolla su labor profesional. Dicho diseño se va configurando de acuerdo con las condiciones, propósitos y hallazgos de la investigación (SÁNCHEZ MACÍAS, 2013).

\section{d) La investigación es un proceso dinámico, simultáneo, imprescindible} al desarrollo de la educación superior. Las materias de investigación, si bien tienen sus tiempos de ejecución no deben cesar sus propósitos en los periodos intermedios entre éstas, por ejemplo, las etapas inter ciclos. Esto se logra mediante la exigencia hacia el alumno de la entrega y presentación de avances al inicio de cada módulo de investigación subsecuente. La continuidad está basada en una revisión de avances al término de la materia y una realimentación acerca de las tareas que deben desarrollarse durante el tiempo que transcurre hasta la siguiente materia de investigación. En el inicio de la siguiente materia se deben entregar y exponer los avances solicitados por el asesor de tesis. El hecho de no estar cursando una materia de investigación no implica que el proceso esté detenido. El compromiso de parte del asesor de tesis con el proceso de investigación es una parte fundamental. Se reconoce que se necesita una gran cantidad de tiempo para revisar, orientar y realimentar todos los trabajos, mucho más del que la institución puede retribuirle monetariamente.

\section{e) El proceso de investigación implica comunicación y socialización de} avances y resultados. Como ya se estableció, las investigaciones que se realizan en educación superior deben buscar impactar positivamente a la sociedad, al contexto del alumno-investigador. Es por eso indispensable que los avances y los resultados sean socializados en varios niveles a saber (ALLEN, EBY, CHAO Y BAURER, 2017):

- Con sus compañeros de grupo.

- Con sus compañeros de programa, en sesiones colegiadas.

- A manera de cartel y/o ponencia en los eventos científicos que organiza la Universidad y los que convoquen instituciones externas. 
- Mediante la publicación de artículos científicos en revistas arbitradas.

Debe ser, además, una práctica imperativa en el aula, que los avances que se presentan al inicio y al final de la materia sean a manera de diálogo-debatereflexión, y valoración crítica, con la presencia de sus compañeros para que apoyen la evaluación desde su carácter de pares (ZILBERSTEIN, SILVESTRE Y OLMEDO, 2016). De ser posible en esta práctica debe estar presente también el Coordinador del Programa y otros profesores, ya que esto establece un ambiente de formalidad adicional que desarrolla competencias en el alumno investigador.

La exposición oral es una de las competencias investigativas que se propone adquiera el alumno-investigador. Su práctica en el aula tiene como ventaja permitirle la experiencia para la formación en varias habilidades (CAHNMANN-TAYLOR Y SIEGESMUND, 2017): hablar ante el público, adaptar la exposición al tipo de audiencia que le escucha, el uso del lenguaje técnicocientífico apropiado, la abstracción de las ideas que se quieren comunicar y su comunicación formal, la sintetización de ideas para adaptarlas al tiempo otorgado para la presentación, entre otras.

La comunicación que se da en el salón de clase debe tener un carácter didáctico, esto implica que el docente conciba como su función primordial la optimización del desarrollo del alumno-investigador mediante el uso de estrategias y técnicas para este fin. Esta postura permite la toma de conciencia de la comunicación como subproceso, es un acto en el cual tanto el profesor como el alumno se identifican entre sí como lo que son, seres humanos interrelacionados en un proceso más complejo de enseñanza aprendizaje (FUENTES, DALDA \& MOREIRA, 2014).

\section{f) El alcance de la tesis debe estar sujeto a la disponibilidad de recursos} del alumno-investigador. El asesor de tesis en su papel de orientadordesarrollador (ZANKOV, 1984, DAVIDOV, 1988) debe propiciar como una actividad preparatoria a la definición de un problema y tema de investigación la realización de un Inventario de recursos para la investigación (ver figura 1.). Dicha práctica implica que el alumno investigador sea consciente de sus recursos antes de emprender el proyecto de investigación. Una desviación en el plan no 


\section{Obutchénie}

debe convertirse en un obstáculo insalvable, sólo la reorientación de la investigación. Una planeación estratégica considera este punto al momento de determinar la delimitación y alcance de la investigación, así como las posibles contingencias que se pueden presentar. Dichos recursos son, a saber:

- Económicos (dinero para materiales, transporte o retribuciones).

- Cognoscitivos (es necesario elegir un tema en el que se utilicen los conocimientos y habilidades que ya se poseen).

- Tiempo (normalmente serían investigaciones de tipo transversal).

- Acceso a la información (en la organización qué datos si se pueden consultar y en su caso divulgar).

- Grado de influencia en el campo de la investigación, que se refiere al grado de autoridad o poder que el alumno investigador tiene en la organización para lograr acceder a recursos y para poder implementar la propuesta. Una mala praxis de este punto lleva a la frustración y desmotivación.

Figura 1 - Inventario de recursos para la investigación

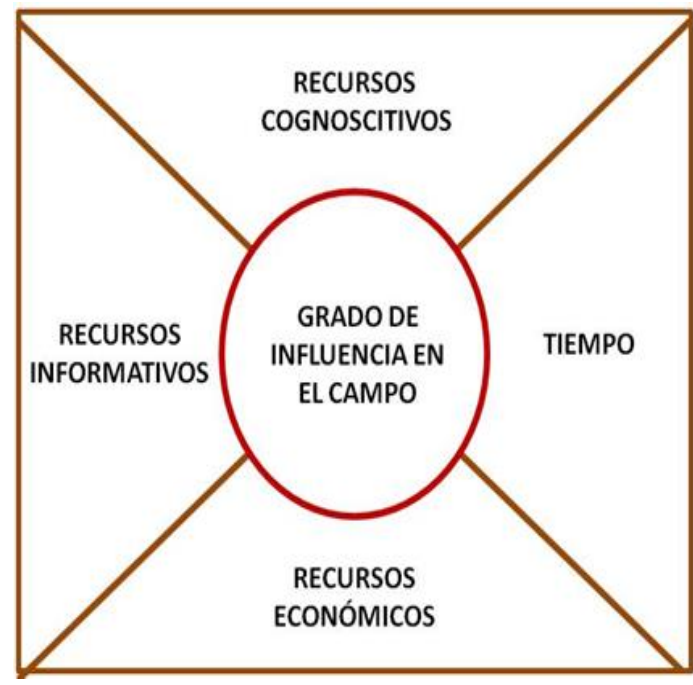

Fuente: Sánchez Macías, 2014

Se considera que este Modelo Pedagógico para la Investigación Administrativa debe orientarse hacia la responsabilidad profesional del egresado, mediante un comportamiento ético, con visión humanista y social. Impulsando el respeto por la verdad científica con un enfoque laico que aporte al bienestar y equidad de la comunidad. El enfoque pedagógico/andragógico 
contempla como principios al aprendizaje activo, significativo y el autoaprendizaje con las siguientes características: ser acumulativo, autogestionado, autorregulado y autónomo, orientado a la meta, situado y colaborativo, así como individualmente diferente.

La educación superior proporciona las competencias complementarias especializadas para el desempeño profesional, pero también las investigativas. En conjunto habilitan al egresado en la generación, desarrollo, adaptación y aplicación del conocimiento. Las estrategias de aprendizaje (SOLIS Y ZILBERSTEIN, 2005), le permiten al estudiante conocer con amplitud y profundidad el estado del conocimiento y del arte, en el área de las Ciencias de la Administración. El Modelo debe promover la investigación aplicada de competencias para la prevención y solución de problemas en las organizaciones en las cuales se desenvuelven los estudiantes. El objetivo último es conseguir un impacto positivo en el entorno y contexto sociocultural mediante el desarrollo del ser humano, en este caso el estudiante.

El contenido a apropiarse por el estudiante (conocimientos, habilidades y valores) debe ser actual y relevante, con profundas bases científicas, y apegado a los nuevos avances y transformaciones de la sociedad, la ciencia, la técnica y el pensamiento humano. Se propone considerar la apropiación del contenido como un proceso en que debe procurarse la implicación del estudiante en tareas de complejidad creciente, de preferencia inter y multidisciplinarias, como vía que desarrolla un pensamiento reflexivo y exige crecimiento en el desempeño como profesional y ser humano comprometido. Estos tipos de tareas implican:

- La búsqueda permanente de la información científica en diversas fuentes (impresas y electrónicas). Se propicia el trabajo en la biblioteca, el uso de libros en formato electrónico, la consulta de la plataforma virtual y el uso de bases de datos científicas.

- La vinculación directa con el área de la Administración en la iniciativa pública o privada.

Los métodos y procedimientos que prevalecen son los que se asemejen a las actividades profesionales de los estudiantes, pero a la vez que implican un 
enfoque histórico cultural entre ellos se privilegian: el estudio de caso, las simulaciones, el aprendizaje basado en la investigación, el aprendizaje basado en problemas, el aprendizaje cooperativo, los proyectos, los trabajos de laboratorio, el diseño de procesos y productos, la elaboración de síntesis de investigaciones, la práctica directa en la producción o los servicios, la elaboración de artículos, la intervención en eventos científicos, entre otros (ZILBERSTEIN, 2004).

El conocimiento por parte del docente de posgrado de la preparación antecedente del estudiante para los nuevos contenidos, su formación profesional anterior y como ser humano. Esto le permite apoyarle en la búsqueda de significado y sentido en lo que aprende, partiendo del diagnóstico de su zona actual de desarrollo y la potenciación de esta (VIGOTSKY, 2013), sobre la base de la interacción en el grupo, en grupos más pequeños, en binas, entre otras formas de organización. Teniendo como base, de preferencia, la solución de problemas profesionales reales del área del conocimiento de que se trate. Por ejemplo, trabajos en equipo acerca de un problema profesional en el que alumno pueda vincular la teoría con la práctica y para ello utilice sus conocimientos previos y del contexto en donde se desenvuelve. Para esto, la planeación de las actividades por parte del docente requiere que este conozca la situación del alumno, sus antecedentes escolares y un diagnóstico inicial para contextualizar los trabajos.

Considerar como condición esencial el vínculo de la teoría con la práctica profesional concreta del Estado, el País y el resto del mundo, en función de conocer y poder aplicar lo que existe en otras partes, pero sin afectar la cultura y desarrollo local, estatal y nacional. Los contenidos de todas las materias deben contribuir a reforzar las líneas de investigación del posgrado y las investigaciones de los estudiantes, siendo esto un requisito indispensable.

El uso de medios o recursos de enseñanza y aprendizaje en correspondencia con los avances más actuales de la sociedad, la ciencia y la técnica. Asumir la evaluación como proceso y resultado, desde una concepción de base humanista, en que la formación de competencias profesionales e investigativas está en el centro de atención del docente, preocupado por el estudiante en cuanto a sus intereses, necesidades, impulso al éxito y por su capacidad de liderazgo. Desde el punto de vista educativo el proceso refuerza la 
formación acorde a la ética de la profesión y el sentido humano del estudiante. A continuación, se presenta el elemento que se considera distintivo en la práctica de la didáctica para la investigación administrativa.

\subsection{El Diagnóstico Organizacional primer eje didáctico de la Investigación administrativa}

La Didáctica de la Investigación Administrativa propuesta asume como sus fuentes a aquellas que permiten conocer por medio de causas o principios las características de una organización y de los fenómenos que ocurren en ella, con rigor y de forma metódica (YACUZZI, 2006) desde un enfoque histórico-lógico. Las fuentes tienen un carácter complementario y se enlistan a continuación:

- Teorías y modelos de los procesos y procedimientos que orientan la acción en la organización.

- El plan estratégico, para contrastar el lugar donde se desea estar y el lugar en el que se encuentra. Así como para comparar lo planeado contra lo realizado.

- El diseño organizacional, su estructura y operacionalización considerando la jerarquía y las relaciones de poder y autoridad vigentes.

- Datos comparativos de las empresas del mismo sector o subsector de la industria y los servicios, también llamado benchmarking (SAAVEDRA GARCÍA Y TAPIA SÁNCHEZ, 2011).

- Comportamientos históricos de la organización.

- La observación sistematizada de los grupos sociales y grupos de interés que integran las organizaciones para acercarse a la realidad desde una percepción colectiva (stakeholders).

De esta manera, aportan la evidencia que describe y/o explica los fenómenos estudiados en la organización, la información que se recoge tiene carácter cuantitativo, pero también cualitativo (GENERO, CRUZ-LEMUS Y PIATTINI, 2014). Lo cual se asume no es una limitante para generar teoría dado que "la contribución de la ciencia social no está en el conocimiento validado, sino más bien en la sugerencia de relaciones y conexiones que hacen cambiar a las acciones y las perspectivas" (WEICK, 1995, p. 387). 
El proceso de investigación propuesto para la Ciencia Administrativa incorpora el uso del Diagnóstico Organizacional como el medio científico para llegar a la concepción del problema a atender mediante el aporte práctico (DI POFI, 2002). Cabe resaltar que la identificación de desviaciones en las organizaciones se logra mediante la investigación y no en base a percepciones o la intuición. No es científico asumir que se puede establecer un problema si no está basado en evidencia obtenida de manera metódica y sistemática.

Se considera al Diagnóstico Organizacional como un método por excelencia en la Investigación Administrativa. Sus raíces etimológicas permiten deducir su significado: día (a través) y gnosis (conocer), conocer a través de o por medio de. Ha sido definido como el proceso de acercamiento gradual al conocimiento analítico de un hecho o problema administrativo que permite destacar los elementos más significativos de una alteración en el desarrollo de las actividades de una institución (LUNA, 2014).

Para Martínez (2010, p. 24) el Diagnóstico Administrativo implica “...el conocimiento mediante el cual se detectan los signos de las desviaciones en la organización. Implica determinación y caracterización de los hechos económicos, administrativos, políticos, culturales y sociales que dominan la realidad sobre la cual se pretende planear."

El Diagnóstico Administrativo (Organizacional) permite conocer los síntomas del equilibrio de la organización; por lo cual se considera como la fase vital de las técnicas de planeación administrativa. En él se evalúan tendencias y situaciones, se explican hechos y se constituyen asociaciones de causalidad relacionadas, además se esclarecen las desviaciones que influyen en una actividad específica (ANSOFF, 1997).

Desde un punto de vista metodológico contiene una caracterización de la organización obtenido a partir de un profundo análisis sistemático de la situación actual y de cómo se llegó a esta, además de una explicación de las causas. "Es un medio para potenciar los recursos y la capacidad estratégica de una organización: es un insumo para la planeación estratégica que reconoce la existencia de un “otro", lo que implica que el entorno es diferente de cero (VIDAL, 2004, p. 20)". Esto está fundamentado en el principio de que la Planeación es un proceso 
dinámico y adaptativo debido que el medio está evolucionando constantemente.

El resultado del Diagnóstico Organizacional desemboca en la Síntesis de la Evaluación del Desempeño Organizacional (SEDO). Una vez que se ha observado, medido, comparado y analizado indicadores e inductores es necesario procesar toda la información obtenida y presentar un resultado que sintetice y relacione de forma lógica toda la información obtenida.

Para identificar, obtener, analizar y sintetizar la información, la ciencia administrativa recurre a varias herramientas de naturaleza cuantitativa y cualitativa, que se presentan a continuación de manera enunciativa y no limitativa y que se consideran como medios indispensables en el modelo de investigación (ver tabla 1.).

Tabla 1 - Categorías para la investigación administrativa.

\begin{tabular}{|c|c|c|}
\hline a) & $\begin{array}{l}\text { El diagnóstico organizacional como } \\
\text { método general en la investigación } \\
\text { administrativa. }\end{array}$ & $\begin{array}{l}\text { - Enfoque sistémico organizacional. } \\
\text { - Enfoque de Administración Estratégica. } \\
\text { - La revisión documental y estadística. } \\
\text { - Identificación y caracterización de stakeholders. } \\
\text { - Diseño de instrumentos (encuestas para masividad, } \\
\text { entrevistas para profundizar, grupos de enfoque, guías de } \\
\text { observación para contraste). }\end{array}$ \\
\hline b) & $\begin{array}{l}\text { Técnicas para diagnóstico y } \\
\text { modelación de propuestas } \\
\text { administrativas. }\end{array}$ & $\begin{array}{l}\text { - El diagrama de Pareto } \\
\text { - El análisis FODA } \\
\text { - Diagrama causa-efecto } \\
\text { - Generación de estrategias mediante el Cruce del FODA } \\
\text { - El Cuadro de Mando Integral } \\
\text { - El Análisis MAPP. }\end{array}$ \\
\hline c) & $\begin{array}{l}\text { Técnicas para la investigación } \\
\text { mercadológica }\end{array}$ & $\begin{array}{l}\text { - Cuestionarios: personales, telefónicos y por internet. } \\
\text { - Entrevistas: estructurada, semi-estructurada, no } \\
\text { estructurada, a profunidad. } \\
\text { - Cliente misterioso (mistery shopper). } \\
\text { - Grupos de enfoque (focus group). } \\
\text { - Evaluación sensorial y escalas hedónicas. }\end{array}$ \\
\hline d) & $\begin{array}{l}\text { Técnicas para la investigación } \\
\text { financiera }\end{array}$ & $\begin{array}{l}\text { - Métodos de análisis financiero: } \\
\text { - Razones financieras. } \\
\text { - Índices de empresa e índices industriales. } \\
\text { - Análisis de proporciones. } \\
\text { - Estimaciones y presupuestos. } \\
\text { - Métodos para análisis de estructura de costos: } \\
\text { - Análisis de costos fijos y variables. } \\
\text { - Análisis de costos de producción y de operación. } \\
\text { - Análisis de sensibilidad. }\end{array}$ \\
\hline
\end{tabular}




\begin{tabular}{|c|c|c|}
\hline & & $\begin{array}{l}\text { - Análisis de grado de apalancamiento operativo y } \\
\text { financiero. }\end{array}$ \\
\hline & $\begin{array}{l}\text { Técnicas para la investigación de } \\
\text { procesos productivos y de } \\
\text { operaciones. }\end{array}$ & $\begin{array}{l}\text { - Líneas flexibles (Hoshin) } \\
\text { - Estándar de trabajo } \\
\text { - Eficacia Global de Equipos (OEE por sus siglas en inglés) } \\
\text { - Modelo de las cinco eses (5s) } \\
\text { - Cambio de matriz en menos de } 10 \text { minutos (SMED por sus } \\
\text { siglas en inglés) } \\
\text { - Sistema Justo a Tiempo (JIT por sus siglas en inglés) } \\
\text { - Sistema de Tarjetas (Kanban) } \\
\text { - Análisis logístico y de flujo de valor } \\
\text { - Seis Sigma (Six sigma) } \\
\text { inglés). }\end{array}$ \\
\hline & $\begin{array}{l}\text { Técnicas para la investigación en } \\
\text { administración del personal y } \\
\text { comportamiento organizacional. }\end{array}$ & $\begin{array}{l}\text { - Test estandarizados para medir actitudes y predecir } \\
\text { comportamientos mediante el uso de escalas } \\
\text { psicométricas. } \\
\text { - Intervenciones para el adiestramiento, capacitación y } \\
\text { desarrollo. } \\
\text { - Instrumentos cualitativos para conocer, analizar y explicar } \\
\text { los fenómenos organizacionales. }\end{array}$ \\
\hline
\end{tabular}

Fuente: Elaboración propia

La lista que se presenta no pretende ser exhaustiva, sin embargo, incluye aquellas metodologías y técnicas administrativas que en algunos casos abrevando de otras ciencias, son usualmente utilizadas en la investigación administrativa.

\subsection{El Diagnóstico Organizacional segundo eje didáctico de la Investigación administrativa}

El concepto de Planeación Estratégica surgió a mediados de los años sesenta gracias a las aportaciones a la Ciencia Administrativa de Ansoff, Chandler y Andrews en la década de los sesentas. "Es un proceso formulado de planeación a largo plazo que se utiliza para definir y alcanzar metas organizacionales" (MINTZBERG, QUINN, Y VOYER, 1997, p. 291).

El objetivo de la Planeación Estratégica (que a últimas fechas ha sido rebautizada como Administración Estratégica para hacer referencia a su implicación en todo el proceso administrativo), es la de obtener el máximo beneficio de los recursos internos, seleccionando de su entorno en dónde y cómo se desplegarán tales recursos para lograr las metas de la organización (HITT Y 
IRELAND, 2017).

La Planeación Estratégica requiere de un análisis del medio ambiente externo e interno el cual puede obtenerse mediante el Diagnóstico Organizacional; y a partir de este punto permite establecer un curso concreto de acción, con los principios que habrán de orientarlo, la secuencia de operaciones y los recursos que serán necesarios. Es por eso, por lo que se considera esta teoría como eje direccionador mediante el cual se puede establecer el trayecto para la propuesta de mejora en la organización que se cristaliza en el aporte práctico de la Investigación Administrativa.

Como es evidente, el Diagnóstico Organizacional considera como objeto y campo de estudio a la organización. El modelo de investigación implica su uso, así como de las herramientas y técnicas que lo constituyen y apoyan. Una vez que se ha establecido y caracterizado de manera científica la problemática es mediante la aplicación de la Planeación Estratégica que se propone el plan a seguir, su implementación y atendiendo al carácter procesal de la investigación se formula una manera de verificar la efectividad del aporte mediante el diseño de algún tipo de control administrativo. En el posgrado, es mediante la aplicación de la propuesta, que se logra la validación lo que da pie al posterior desarrollo del aporte teórico.

La integración de la secuencia para la Investigación Administrativa mediante el uso del Diagnóstico Organizacional y con enfoque de Planeación Estratégica se muestra en la figura 2. Cabe resaltar que este proceso es sólo una parte integrante del modelo didáctico que se presenta en la figura 3. 
Figura 2 - Secuencia para la Investigación Administrativa mediante el uso del Diagnóstico Organizacional y con enfoque de Planeación Estratégica

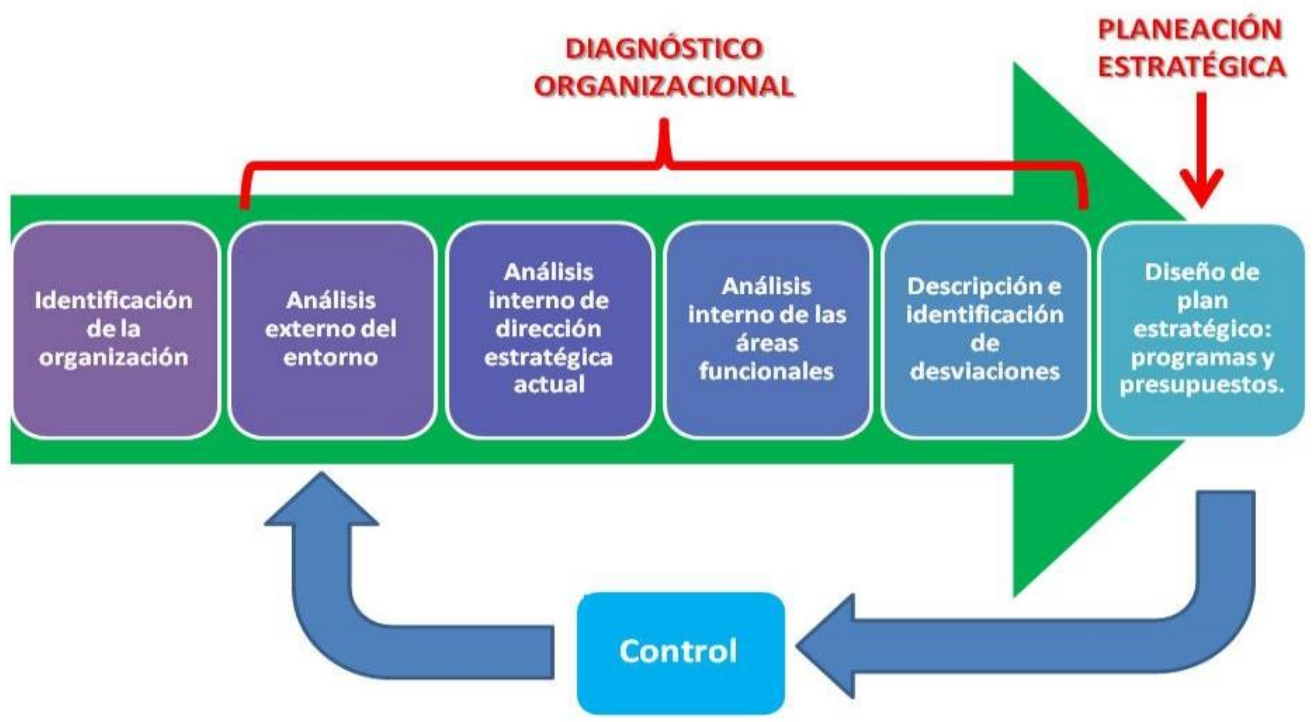

Fuente: Sánchez Macías, 2014

Considerando la secuencia que se propone, es claro que un estudiante de educación superior, generalmente principiante en la investigación debe fundamentar teóricamente las herramientas que utilizará para realizar el diagnóstico organizacional (que en su mayoría implicará investigación empírica) y a la vez, una vez realizado este e identificado la problemática a atender, será necesario volver a documentarse para poder sustentar con teoría la propuesta de mejora que se propone.

En la figura 2 se observa este proceso desde el diseño de la investigación (que se convierte posteriormente en la introducción de la tesis) hasta el arribo al aporte práctico como finalidad del proyecto de tesis y que impacte en la práctica de las organizaciones. Se observan también los momentos en que interviene la investigación documental y la empírica durante el proceso. Cabe resaltar que el autor no considera que un solo tipo de investigación (empírica o documental) que aparece en la figura 3 corresponde a un momento determinado, pero sí se asume que es predominante. 
Figura 3 - Papel de los tipos de investigación para fundamentar teóricamente las acciones a realizar durante el proceso

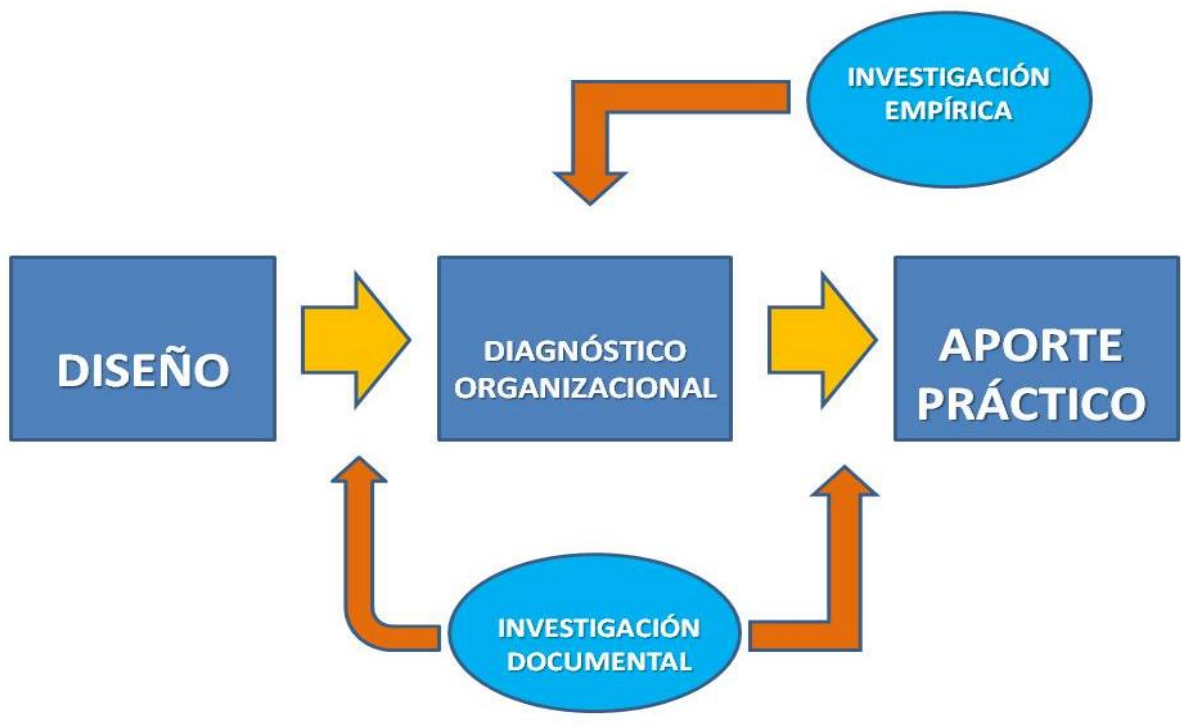

Fuente: Sánchez Macías, 2014

El Modelo investigado considera como ejes de la Investigación Administrativa el estudio de organizaciones existentes para analizarlas y establecer su situación actual; identificando desviaciones y proponiendo mejoras a su estructura o a sus procesos desde su contexto histórico cultural. Sin embargo, se considera también la proyección de nuevas organizaciones que aún no han sido constituidas, desde la labor de la generación de planes de negocios que fundamenten con estudios mercadológicos, técnicos, organizacionales y económicos su viabilidad, para en su momento, estar en posibilidad de solicitar financiamiento a potenciales socios o a instituciones financieras públicas o privadas para llevarla a la realidad.

Esto es así porque la visión empresarial y el espíritu emprendedor son un elemento fundamental en el enfoque pedagógico de la Universidad dada su capacidad de promover la generación de empleos y el bienestar económico. Asimismo, con una fuente de autoempleo que propicie el desarrollo sustentable y el equilibrio de la dimensión social, ecológica y económica (PREMAND, BRODMANN, ALMEIDA, GRUN Y BAROUNI, 2016). 


\section{Conclusiones}

Ante los grandes retos que se le presentan a México en las dimensiones económica y social las IES deben asumir de manera plena el compromiso y responsabilidad de convertirse en formadores de recursos humanos comprometidos con la sociedad y su país. Para las universidades el desafío es continuar creciendo y elevando su calidad, trascender su propio carácter vinculando la investigación a las necesidades sociales concretas.

Es en la educación superior donde se prepara a los profesionales para interpretar al medio ambiente y desarrollar proyectos y cursos de acción viables que propicien la productividad y el desarrollo de las organizaciones. La incorporación de la investigación a las carreras universitarias es indispensable en la formación de estudiantes universitarios, si lo que se desea es educar por encima de adiestrar. El pensamiento teórico y reflexivo, basado en la mayor cantidad de opiniones, posturas y fuentes está muy por encima de la estandarización de la actuación profesional y su visión instrumentalista. El desarrollo sostenible en la visión empresarial pasa necesariamente por la mente de profesionales competentes que han recibido una educación que ve más allá que los intereses particulares o de grupo, con una perspectiva social-humanística.

\section{Referências}

ACOSTA, A. (2014). El futuro de la educación superior en México. Revista Iberoamericana de Educación Superior, V (13), 91-100. Disponible en:<http://www.redalyc.org/articulo.oa?id=299130713006> ISSN

ALLEN, T. D., EBY, L. T., CHAO, G. T., y BAUER, T. N. (2017). Taking stock of two relational aspects of organizational life: Tracing the history and shaping the future of socialization and mentoring research. Journal of Applied Psychology, 102(3), 324-337.

ANSOFF, H. I. (1997). Strategic Management. New York: Palgrave Macmillan.

BOISIER, S. (2016). Sociedad del conocimiento, conocimiento social y gestión territorial. Interações (Campo doi:http://dx.doi.org/10.20435/interações.v2i3.583

Grande), 2(3). 
CAHNMANN-TAYLOR, M. y SIEGESMUND, R. (2018). Arts-Based Research in Education: Foundations for Practice, 2nd edition. New York: Taylor \& Francis. ISBN: 9781315305059.

DAVIDOV. V, (1988). La enseñanza escolar y el desarrollo psíquico. Moscú: Editorial Progreso.

DI POFI, J. A. (2002). Organizational diagnostics: integrating qualitative and quantitative methodology, Journal of Organizational Change Management, Vol. 15 Issue: 2, pp.156-168, https://doi.org/10.1108/09534810210423053

DIDRIKSSON, A. (2004). La Universidad en la Producción Moderna del Conocimiento. En A. Didriksson. Retos y paradigmas: el futuro de la educación superior en México (págs. 23-70). Ciudad de México: Plaza y Valdés.

DÍAZ, A., y HERNÁNDEZ, R. (2015). Constructivismo y aprendizaje significativo. Género, M., Cruz-Lemus, J. A. y Piattini, M. G. (2014). Métodos de investigación en ingeniería de software. Madrid: Ra-Ma.

FUENTES, M. D., DALDA, B., \& MOREIRA, E. (05 de 09 de 2014). El Docente y la Comunicación didáctica en el proceso de enseñanza-aprendizaje. Obtenido de X Taller Regional del Centro de Estudios e Investigación Educacional. Universidad de ciencias pedagogicas. "manuel ascunce domenech."Centro de Estudios e Investigación $\quad$ Educacional.: http://www.cubaeduca.cu/el-docente-y-lacomunicacion-didactica-en-el-proceso-de-ensenanza-aprendizaje

GUTIÉRREZ, M. D. (1992). Educación y valores. Educación y valores en España (p. 98-103). Cádiz: Ministerio de Educación.

HITT, M. y IRELAND, R. D. (2017). The Intersection of Entrepreneurship and Strategic Management Research. Toronto: Wiley. Online ISBN:9781405164214 |DOI:10.1002/9781405164214. http://dx.doi.org/10.1037/apl0000086

LEONTIEV, A. (1972). Problemas del desarrollo del la psiquis. Universidad Estatal de Moscú. Moscú.

LEONTIEV, A. (1982). Actividad, Conciencia y Personalidad. Primera Reimpresión. Pueblo y Educación. La Habana. Cuba.

LUNA, A. C. (2014). Administración estratégica. Ciudad de México: Grupo Editorial Patria. ISBN: 9786074388893.

MARTÍneZ, V. M. (2010). Diagnóstico Administrativo Holístico. Ciudad de México: Trillas. ISBN: 978-6071704139.

MARTÍNEZ-OTERO, V. (2007). La buena educación: reflexiones y propuestas de psicopedagogía humanista. Barcelona: Anthropos Editorial. 
MINTZBERG, HENRY, QUINN, JAMES B. y VOYER, J. (1997). El proceso estratégico: conceptos, contextos y casos. México: Pearson Educación. ISBN: 9789688808290

ORTEGA Y GASSET, J. (2004). Obras completas, Vol. I. Madrid: Taurus.

PREMAND, P., BRODMANN, S., ALMEIDA, R., GRUN, R. y BAROUNI, M., (2016) Entrepreneurship Education and Entry into Self-Employment Among University Graduates, World Development, Volume 77, 2016, Pages 311-327, ISSN 0305-750X, https://doi.org/10.1016/j.worlddev.2015.08.028.

SAAVEDRA GARCÍA, M. L. y TAPIA SÁNCHEZ, B. (2011). Mejores Prácticas y Factores de Competitividad en las Micro, Pequeñas y Medianas Empresas Mexicanas. Economía [en línea] 2011, (Julio-Diciembre) : [Fecha de consulta: 10 de julio de 2018] Disponible en:<http://www.redalyc.org/articulo.oa?id=195623319002> ISSN 1315-2467

SÁNCHEZ MACÍAS, A. (2013) Estrategia Didáctica para orientar la elaboración del Marco Teórico en una Investigación del área de las Ciencias Sociales. Atenas [en linea] 2013, 3 [Fecha de consulta: 10 de julio de 2018] Disponible en:<http://www.redalyc.org/articulo.oa?id=478048959002> ISSN

SÁNCHEZ MACÍAS, A. (2014). Modelo para la investigación en el área de Administración del Posgrado de la Universidad Tangamanga. Tesis doctoral. Tlaxcala: Universidad de Tlaxcala.

SILVESTRE, M y ZILBERSTEIN, J. (2004). Didáctica desarrolladora desde el Enfoque Histórico Cultural. México: CEIDE.

SOLIS, Y. y ZILBERSTEIN, J. (2005). Las estrategias de aprendizaje comprendidas desde el Enfoque Histórico Cultural. México: CEIDE.

VIDAL, E. (2004). Diagnóstico organizacional, evaluación sistemática del desempeño empresarial en la era digital. Colombia: Editorial ECOE Ediciones.

VIGOTSKY, L. (2013). Pensamiento y lenguaje. Madrid: Paidós.

WEICK, K. E. (1995). What theory is not, theorizing is. Administrative Science Quarterly, 1995: Vol. 40, Septiembre, pp. 385-390.

YACUZZI, E. (2006). El estudio de caso como metodología de investigación: teoría, mecanismos causales, validación. CEMA, 2006: 1-37. Disponible en [http://www.ucema.edu.ar/publicaciones/download/documentos/296.pdf]

ZANKOV, L. (1984). La enseñanza y el desarrollo, Moscú. Editorial Progreso. 
ZILBERSTEIN, J. (2004). Aprendizaje, enseñanza y Educación Desarrolladora. México: CEIDE

ZILBERSTEIN, J. (2004). Necesidad de establecer Principios Didácticos en un Proceso de Enseñanza Aprendizaje Desarrollador. En M. Silvestre, \& J. Zilberstein, Didáctica desarrolladora desde el enfoque histórico cultural. (págs. 102-109). México: CEIDE.

ZILBERSTEIN, J. y OLMEDO, S. (2014) Las estrategias de aprendizaje desde una didáctica desarrolladora. Atenas [en linea] 2014, 3 (Julio-Septiembre) : [Fecha de consulta: 10 de julio de 2018] Disponible en: $<$ http://www.redalyc.org/articulo.oa?id=478047203004> ISSN

ZILBERSTEIN, J. y OLMEDO, S. (2015). Didáctica desarrolladora: posición desde el enfoque histórico cultural. Educação e Filosofia, v. 29, n. 57 Disponible en [http://dx.doi.org/10.14393/REVEDFIL]. issn.0102-6801.v29n57a2015-p61a93.

ZILBERSTEIN, J. SILVESTRE, M. y OLMEDO, S. (2016). Diagnóstico y Transformación de la Institución Docente. México: CEIDE.

\section{Armando Sánchez-Macías}

Doctor en Educación. Profesor Investigador de la Coordinación Académica Región Altiplano Oeste. Universidad Autónoma de San Luis Potosí, México. armando.sanchez@uaslp.mx

\section{José Zilberstein Toruncha}

Doctor en Ciencias Pedagógicas. Director del Campus Tequis de la Universidad Tangamanga, México.

jzilberstein@utan.edu.mx

\section{Virginia Azuara Pugliese}

Doctora en Mercadotecnia. Coordinadora de la Licenciatura en Administración en la Coordinación Académica Región Altiplano Oeste. Universidad Autónoma de San Luis Potosí, México. virginia.azuara@uaslp.mx 